\title{
Autonomic function in subjects with severe COPD
}

\author{
Jibril Mohammed ${ }^{1,2}$, Eric Derom ${ }^{3,4}$, Tine De Backer ${ }^{3}$, Jessica Van Oosterwijck ${ }^{1,5}$, Bihiyga Salhi ${ }^{3,4}$, Hellen Da Silva ${ }^{1}$, Patrick Calders ${ }^{1}$
}

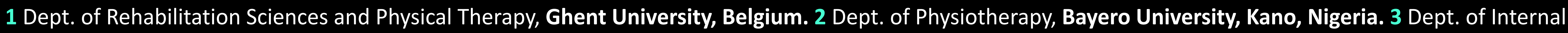

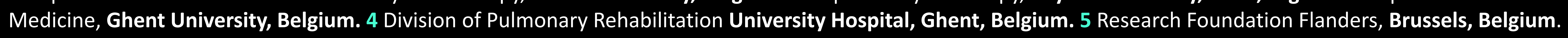

\section{Background and aim}

Patients with COPD show impairments in several extra-pulmonary systems such as the cardiovascular and autonomic nervous systems(ANS).

Autonomic function(AF) parameters involves heart rate variability (HRV) and baroreceptor reflex sensitivity(BRS). And the assessment of these AF parameters have been the focal point in understanding the impairments in the ANS in COPD. Furthermore, cardiac autonomic regulation is known to be influenced by several variables including disease status, AF test protocol and various patient characteristics.

Therefore, this study assessed AF using a standardized test protocol in a cohort of non-sedentary individuals with severe COPD

\section{Contact details}

email: jibril.mohammed@ugent.be

\section{Methods}

26 severe COPD (65 \pm 9 years; BMI: $26 \pm 3.5 \mathrm{~kg} / \mathrm{m}^{2} ; \%$ predictedFEV $\left._{1}: 39.5\right)$ and 21 healthy age-matched control subjects (64 \pm 8years; BMl: $\left.25 \pm 2.8 \mathrm{~kg} / \mathrm{m}^{2}\right)$ participated in this study.

Continuous ECG monitoring was conducted during rest (15 minutes), deep breathing (6 cycles/minute), valsalva maneuver and tilting (to $60^{\circ}$ ) from the supine position.

Main outcomes:

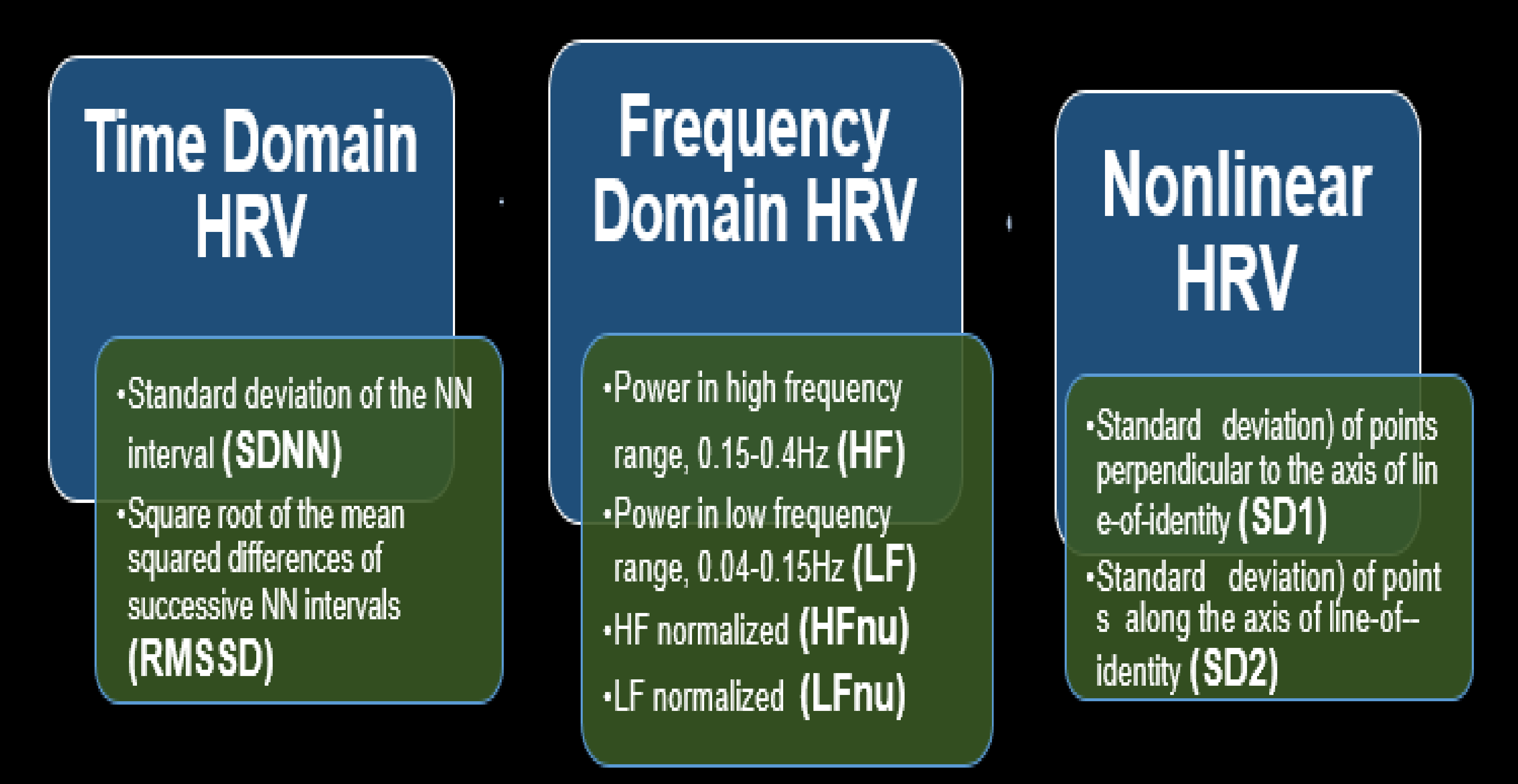

\section{Acknowledgements}

Jibril Mohammed is a PhD student funded by TETFund, Nigeria. Jessica Van Oosterwijck is a postdoctoral research fellow funded by the FWO, Brussels, Belgium.

\section{Results}

The results indicated that both COPD and controls showed comparable values for resting HRV, heart response to deep breathing, Valsalva maneuver parameters and the BRS parameters ( $p>0.05)$. However, following the tilting, the results indicated that subjects with COPD had significantly lower HRV values compared to control group subjects $(p<0.05)$.

Table 1: Results of HRV after tilting in COPD vs controls

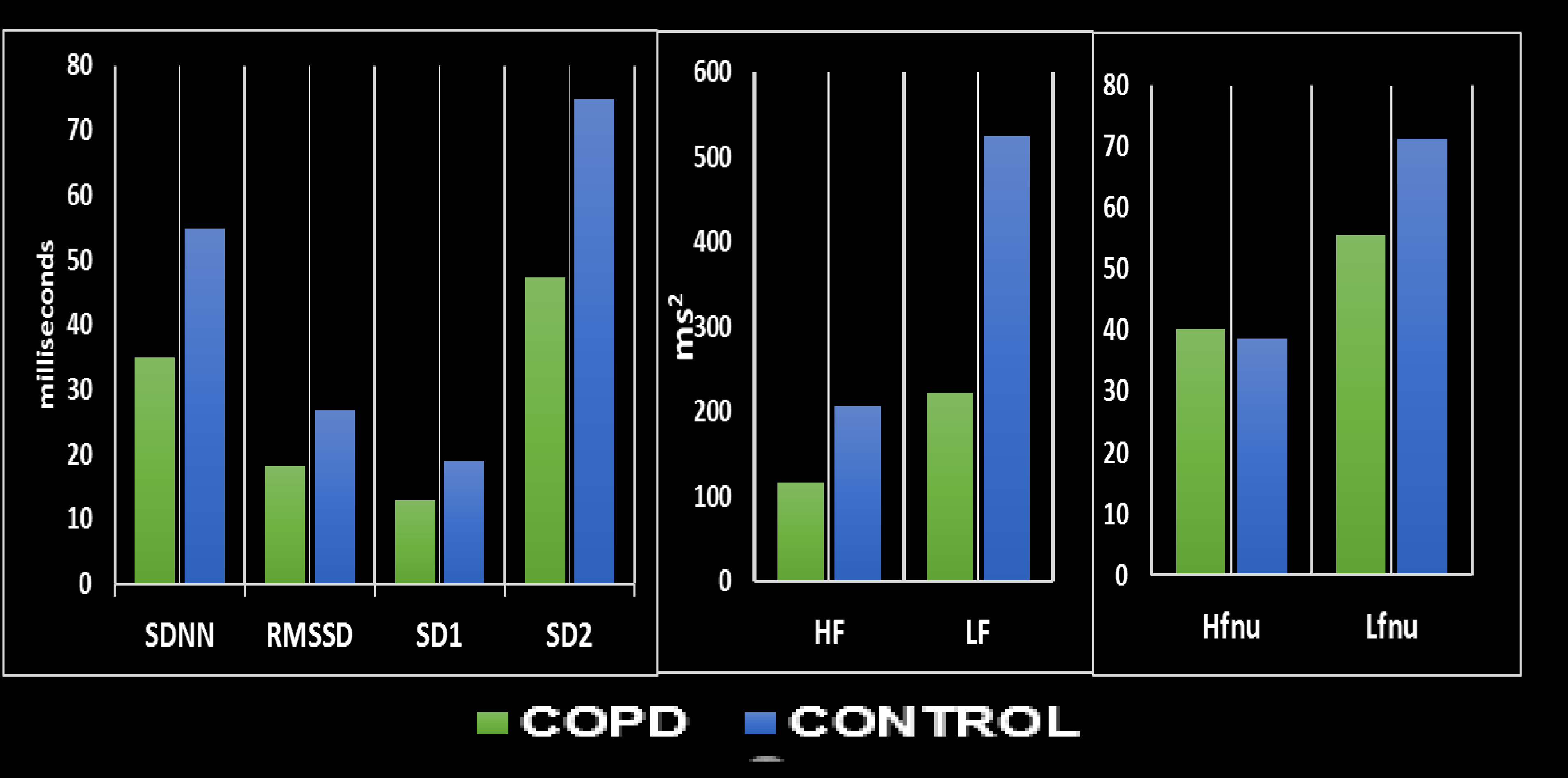

\section{Conclusion}

These results suggest that certain ANS deficits exist in non-sedentary COPD patients compared to healthy controls. 